\title{
THE EFFECT OF STRATIFICATION ON THE AERODYNAMIC ROUGHNESS LENGTH AND DISPLACEMENT HEIGHT
}

\author{
Sergej S. Zilitinkevich ${ }^{1 \star \dagger}$, Ivan Mammarella**, Alexander A. Baklanov ${ }^{\ddagger}$, Sylvain M. Joffre* \\ * Finnish Meteorological Institute, Helsinki, Finland; ${ }^{* *}$ Dep. of Physics, University of Helsinki, Finland; ${ }^{\ddagger}$ Danish \\ Meteorological Institute, Copenhagen, Denmark; ${ }^{\dagger}$ Nansen Environmental and Remote Sensing Centre, Bergen, \\ Norway
}

\section{ABSTRACT}

The roughness length, $z_{0 u}$, and displacement height, $d_{0 u}$, characterise the resistance exerted by the roughness elements on turbulent flows and provide a conventional boundary condition for a wide range of turbulent-flow problems. Classical laboratory experiments and theories treat $z_{0 u}$ and $d_{0 u}$ as geometric parameters independent of the characteristics of the flow. In this paper, we demonstrate essential stability dependences - stronger for the roughness length (especially in stable stratification) and weaker but still pronounced for the displacement height. We develop a scaling-analysis model for these dependences and verify it against experimental data.

\section{INTRODUCTION}

The concepts of the roughness length, $z_{0 u}$, and displacement height, $d_{0 u}$, were introduced in the early thirties to parameterize the transfer of momentum from turbulent flows to aerodynamically rough surfaces, i.e. those with typical roughness-element heights, $h_{0}$, larger than the viscous layer height, $v / u_{*}$, where $v$ is the molecular viscosity and $u_{*}$ is the friction velocity. Laboratory experiments in neutrally stratified boundary-layer flows have shown that roughness parameters do not depend on the characteristics of the flow and factually represent geometric characteristics of the surface. We show that this universally accepted conclusion, is not quite correct; develop new formulations accounting for the effect of stratification on $z_{0 u}$ and $d_{0 u}$; and demonstrate that this effect is generally strong and practically important.

We consider the atmospheric boundary layer $(A B L)$ over a horizontally homogeneous surface covered with obstacles with the typical height $h_{0}$. In neutral stratification, in the so-called "surface layer" (that is at heights $z$ exceeding $\sim 1.6 h_{0}$ but much smaller than the ABL height, $h$ ) the locally generated turbulence does not depend neither on $h$ nor on the characteristics of the surface and, therefore, is fully characterised by only two parameters: the height over the surface, $z$, and the friction velocity, $u_{*}$. Then the eddy viscosity, $K_{M}$, and the velocity gradient, $\partial U / \partial z$, are

$$
K_{M}=k u_{*} z, \quad \frac{\partial U}{\partial z}=\frac{\tau}{K_{M}}=\frac{u_{*}}{k z}
$$

where $k \cong 0.4$ is the von Karman constant. Integrating the second formula in Eq. (2) includes a constant of integration: $U=k^{-1} u_{*} \ln z+$ constant, or equivalently:

$$
U=\left(\frac{u_{*}}{k}\right) \ln \frac{z}{\mathrm{z}_{0 u}} \quad \text { or, more generally, } \quad U=\left(\frac{u_{*}}{k}\right) \ln \frac{z-d_{0 u}}{\mathrm{z}_{0 u}},
$$

where $z_{0 u}$ is just the roughness length (a redefined constant of integration), and $d_{0 u}$ is the displacement height - both measured in length units [see Section 5.4 in Monin and Yaglom (1971), Chapter 4 in Garratt (1992), and references therein].

The downward transfer of momentum over rough surfaces is performed basically by the pressure forces caused by the flow-obstacle interactions and is characterised by the roughness-layer velocity scale $U_{R} \sim u_{*}$ and the geometry of roughness elements, primarily, the roughness-layer height scale, $h_{0}$. For roughness elements of standard shape separated by standard distances, it is reasonable to assume that $z_{0 u}$ and $d_{0 u}$ depend only on

\footnotetext{
${ }^{1}$ Corresponding author: sergej.zilitinkevich@fmi.fi
} 
the above two parameters $\left(h_{0}\right.$ and $u_{*}$ ), which immediately yields $z_{0 u} \sim h_{0}$ and $d_{0 u} \sim h_{0}\left(u_{*}\right.$ drops out for dimensional reasons). Classical experiments with sand roughness confirmed this conclusion and gave $z_{0 u}=$ $h_{0} / 30$, whereas experiments with very rough surfaces gave the typical value of $d_{0 u}=2 h_{0} / 3$ (e.g., Monin and Yaglom, 1971; Garratt, 1992).

Generally $z_{0 u} / h_{0}$ and $d_{0 u} / h_{0}$ depend on the shape and area density of the roughness elements. Accordingly, land surfaces are characterised by their standard roughness lengths and displacement heights - usually considered as geometric parameters independent of the wind speed and stratification. Since the 1970s, evidence has appeared that $z_{0 u}$ may depend on the stratification, but the traditional consensus about $z_{0 u}$ as a geometric parameter was practically not shaken and, to the best of the authors' knowledge, no systematic quantitative analyses of the stratification effects on $z_{0 u}$ and $d_{0 u}$ have been performed.

\section{THEORETICAL MODEL}

The vertical length scale characterising the effect of stratification close to the surface is the Obukhov length (Monin and Obukhov, 1954):

$$
L=-u_{*}^{3} / F_{b}
$$

where $F_{b}$ is the vertical turbulent flux of buoyancy, $b$, defined as $b=g \rho / \rho_{0}$ ( $\rho$ is fluid density, $\rho_{0}$ is its reference value, and $g$ is the acceleration of gravity). In the atmosphere, neglecting the effect of humidity, $F_{b}=$ $(g / T) F_{\theta}$, where $T$ is a reference value of the absolute temperature, and $F_{\theta}$ (negative in stable and positive in unstable stratification) is the vertical turbulent flux of potential temperature, $\Theta$. As a general rule, the role of stratification is negligible at $z<<I L I$, but becomes crucial at $z \geq I L I$ (Monin and Yaglom, 1971). Then, recalling that ILI often approaches $20-30 \mathrm{~m}$, it is only natural to expect an essential effect of stratification on the roughnesslayer turbulence and therefore on $z_{0 u}$ and $d_{0 u}$ for urban or woodland surfaces with $h_{0} \sim 20 \mathrm{~m}$ or larger.

Physically $z_{0 u}$ is not a geometric feature of the surface, but the depth scale of a sub-layer within the roughness layer corresponding to the velocity decrease from its maximal value, $U_{R}$, approached at the canopy's upper boundary, $z \sim h_{0}$, to its minor fraction, say $10 \%$ of $U_{R}$, somewhere within the canopy layer. This definition allows the estimation of $z_{0 u}$ through the roughness-layer eddy viscosity scale, $K_{M 0}$ (Zilitinkevich et al., 2008):

$$
z_{0 u} \sim K_{M 0} / u_{*}
$$

whereas scaling estimates of $K_{M 0}$ relevant to different regimes can be obtained by matching the roughness layer, $0<z<1.6 h_{0}$, with the surface layer, $1.6 h_{0}<z<0.1 h$, which yields the following roughness length formulations for stable $(L>0)$ and unstable $(L<0)$ stratifications:

$$
\frac{z_{0 u}}{z_{0}}=\frac{1}{1+C_{Z S} h_{0} / L} \text { for } L>0, \quad \frac{z_{0 u}}{z_{0}}=1+C_{Z C}\left(\frac{h_{0}}{-L}\right)^{1 / 3} \text { for } L<0 \text {, }
$$

where $C_{Z S}$ and $C_{Z C}$ are dimensionless constants to be determined empirically.

The displacement height, $d_{0 u}$, is the depth scale of a lower, stagnated part of the roughness layer, where the mean wind is so weak that the momentum transfer from the air flow to the roughness elements can be neglected. The larger the well-ventilated fraction of the roughness layer $\left(\sim z_{0 u} / h_{0}\right)$ the smaller should be its stagnated share $\left(\sim d_{0 u} / h_{0}\right)$, suggesting a monotonic decrease of $d_{0 u} / h_{0}$ with a decrease in $h_{0} / L$ (that is decreasing stability or increasing instability). On this basis we derived the following formulae 


$$
d_{0 u}=d_{0}+\left(h_{0}-d_{0}\right) \frac{h_{0} / L}{C_{D S}+h_{0} / L} \quad \text { for } L>0 ; d_{0 u}=\frac{d_{0}}{1+C_{D C}\left(-h_{0} / L\right)^{1 / 3}} \quad \text { for } L<0
$$

where $C_{D S}$ and $C_{D C}$ are dimensionless coefficients.

\section{VERIFICATION AND DISCUSSION}

The above results have been verified against data from the mean-profile and turbulence measurements of the wind speed, $U$, potential temperature, $\Theta$, friction velocity, $u_{*}$ (determined as the square root of the vertical flux of momentum), and the vertical flux of potential temperature, $F_{\theta}$ (and hence $L=-u_{*}^{3} / \beta F_{\theta}$ ). For the stable scarification, we used data from a 48-m tower obtained during July 2003 - June 2004 over a boreal forest at the Sodankylä Meteorological Observatory, $100 \mathrm{~km}$ north of the Arctic Polar Circle (Joffre et al., 2001; Gryning et al., 2001); and for the unstable stratification, data from the "Basel-Sperrstrasse" 32-m meteorological tower (dataset BUBBLE = Basel Urban Boundary Layer Experiment, 2001-2002, Rotach et al., 2005), excluding obviously irrelevant situations, such as those with winds blowing along street canyons. Data analyses confirm our theoretical model, Eqs. (5)-(6) and yield the following values of the empirical constants: $C_{Z S}=8.13, C_{Z C}=1.15$, $C_{D S}=1.05, C_{D C}=0.56$.

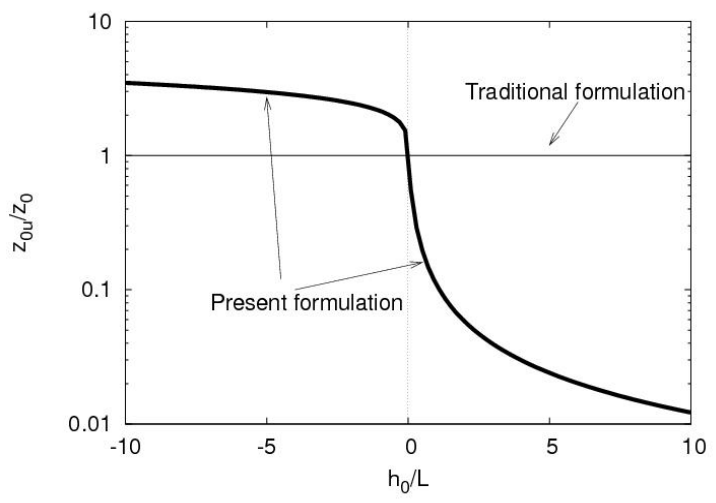

Figure 1. The stability dependence of the roughness length: $z_{0 u}$, normalised by its neutralstability value, $z_{0}$, is shown as dependent on $h_{0} / L$, where $h_{0}$ is the typical height of the roughness elements, and $L=-u_{*}^{3}\left(\beta F_{\theta s}\right)^{-1}$ is the Monin-Obukhov length scale.

Figure 1 shows $z_{0 u} / z_{0}$ versus $h_{0} / L$ after Eq. (5) with $C_{Z S}=8.13$ and $C_{Z C}=1.15: z_{0 u}$ monotonically decreases with increasing stability and, in the meteorological interval $-10<h_{0} / L<10$, varies over more than two orders of magnitude, from $4 z_{0}$ to $10^{-2} z_{0}$. In the resistance law, expressing the friction velocity, $u_{*}$, through the mean wind velocity, $U\left(z_{1}\right)$, at a given level, $z=z_{1}$ (the lowest computational level in atmospheric models), $z_{0 u}$ appears in combinations $\ln \left(z_{1} / z_{0 u}\right)$ or $\ln \left[\left(z_{1}-d_{0 u}\right) / z_{0 u}\right]$. Then, overestimation of $z_{0 u}$ by two orders of magnitude (at $h_{0} / L \sim 5$ ) causes a few times overestimation of $u_{*}$.

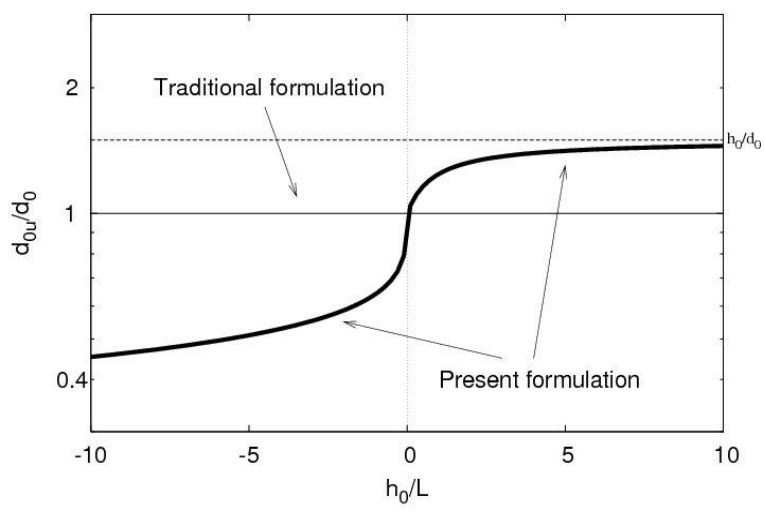

Figure 2. The stability dependence of the displacement height: $d_{0 u}$, normalised by its neutral-stability value, $d_{0}$, is shown as dependent on $h_{0} / L$. 
Figure 2 shows $d_{0 u} / d_{0}$ versus $h_{0} / L$ after Eq. (6) with $C_{D S}=1.05$ and $C_{D C}=0.56: d_{0 u}$ increases from $d_{0} / 2 \sim h_{0} / 3$ in strong convection to $3 d_{0} / 2 \sim h_{0}$ in strongly stable stratification. In the numerical weather prediction (NWP) and climate modelling applications this effect is almost negligible as $z_{1}$ is usually quite large (e.g., $z_{1} \approx 30 \mathrm{~m}$ in the NWP model system HIRLAM), so that $z_{1}-\left(h_{0} / 3\right)$ and $z_{1}-h_{0}$ differ only slightly. However, $d_{0 u}$ represents a measure of the depth of the lower, stagnated part of the urban or vegetation canopy layer. In this capacity $d_{0 u}$ is quite important as such, especially in the air quality applications, and its threefold variability shown in Figure 2 deserves attention.

\section{CONCLUSIONS}

The above essential features of the flow-surface interaction over very rough surfaces in extreme stratification regimes are to be taken into account to refine current formulations of the drag coefficient $C_{d}=u_{*} / U_{1}$, where $U_{1}$ is the wind speed at the reference height $z_{1}$. As follows from our analysis, the dependence of $C_{d}$ on the static stability is caused by the two factors: the $z / L$ effect on the surface-layer velocity profile, and the $h_{0} / L$ effect on the roughness length and displacement height [Eqs. (5) and (6)]. Until recently the role of the second factor was overlooked.

In our approach, the geometry of the roughness elements (in particular, the typical distance between them) is reflected in the ratio between the neutral-stability roughness length, $z_{0}$, and the roughness element height, $h_{0}$. Recall that $z_{0} / h_{0}$ is a strongly variable parameter comprehensively investigated within the traditional approach. In this paper we base on the already known $z_{0} / h_{0}$ and extend the traditional concept accounting for the stability dependences of $z_{0 u} / z_{0}$ and $d_{0 u} / d_{0}$.

\section{ACKNOWLEDGEMENTS}

This work has been supported by the EU FP7 projects PBL-PMES (Grant agreement No. 227915) and MEGAPOLI (No. 212520); and the Academy of Finland Project IS4FIRES.

\section{References}

Garratt J.R. 1992. The atmospheric boundary layer. Cambridge University Press, Cambridge, U. K., 316 pp.

Gryning S.E., Batchvarova E., De Bruin H.A.R. 2001. Energy balance of a sparse coniferous high-latitude forest under winter conditions. Boundary-Layer Meteorol. 99: 465-488

Joffre S.M., Kangas M., Heikinheimo M., Kitaigorodskii S.A. 2001. Variability of the stable and unstable atmospheric boundary-layer height and its scales over a boreal forest. Boundary-Layer Meteorol. 99: 429450

Monin A.S., Obukhov A.M. 1954. Basic laws of turbulence mixing in the surface layer of the atmosphere. Trudy Geofiz. Inst. AN SSSR 24 (151): 163-187

Monin A.S., Yaglom A.M. 1971. Statistical Fluid Mechanics, Vol. 1. MIT Press, Cambridge, Massachusetts, and London, England, $769 \mathrm{pp}$.

Rotach M.W., et al. 2005. BUBBLE - an Urban Boundary Layer Meteorology Project. Theor. Appl. Climatol. 81: 231-261

Zilitinkevich S.S., Mammarella, I., Baklanov, A.A., Joffre, S.M. 2008. The effect of stratification on the aerodynamic roughness length and displacement height. Boundary-Layer Meteorol. 129: 179-190. 